Article

\title{
In Vitro Anti-Inflammatory Activity in Arthritic Synoviocytes of A. brachypoda Root Extracts and Its Unusual Dimeric Flavonoids
}

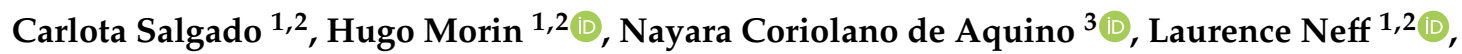

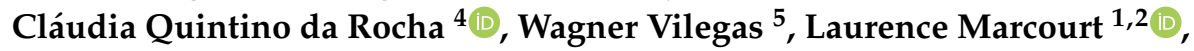 \\ Jean-Luc Wolfender ${ }^{1,2}$, Olivier Jordan 1,2 ${ }^{-1}$, Emerson Ferreira Queiroz ${ }^{1,2}$ \\ and Eric Allémann 1,2,*(D) \\ 1 School of Pharmaceutical Sciences, University of Geneva, 1211 Geneva-4, Switzerland; \\ carlota.salgado@unige.ch (C.S.); hugo.morin@unige.ch (H.M.); laurence.neff@unige.ch (L.N.); \\ laurence.marcourt@unige.ch (L.M.); jean-luc.wolfender@unige.ch (J.-L.W.); olivier.jordan@unige.ch (O.J.); \\ emerson.ferreira@unige.ch (E.F.Q.) \\ 2 Institute of Pharmaceutical Sciences of Western Switzerland, University of Geneva, \\ 1211 Geneva-4, Switzerland \\ 3 Departamento de Química Orgânica e Inorgânica, Universidade Federal do Ceará, \\ 60450-765 Fortaleza-CE, Brazil; nayaracoriolano@hotmail.com \\ 4 Laboratório de Produtos Naturais, Centro de Ciência Exatas e Tecnologia, Departamento de Química, \\ 65080-805 São Luís-MA, Brazil; claudiarocha3@yahoo.com.br \\ 5 Experimental Campus of the Paulista Coast, UNESP-São Paulo State University, \\ 11330-900 São Vicente-SP, Brazil; vilegasw@clp.unesp.br \\ * Correspondence: eric.allemann@unige.ch
}

Received: 5 October 2020; Accepted: 5 November 2020; Published: 9 November 2020

\begin{abstract}
Arrabidaea brachypoda is a plant commonly used for the treatment of kidney stones, arthritis and pain in traditional Brazilian medicine. Different in vitro and in vivo activities, ranging from antinociceptive to anti-Trypanosoma cruzi, have been reported for the dichloromethane root extract of Arrabidaea brachypoda (DCMAB) and isolated compounds. This work aimed to assess the in vitro anti-inflammatory activity in arthritic synoviocytes of the DCMAB, the hydroethanolic extract (HEAB) and three dimeric flavonoids isolated from the DCMAB. These compounds, brachydin A (1), B (2) and C (3), were isolated both by medium pressure liquid and high-speed counter current chromatography. Their quantification was performed by mass spectrometry on both DCMAB and HEAB. IL-1 $\beta$ activated human fibroblast-like synoviocytes were incubated with both extracts and isolated compounds to determine the levels of pro-inflammatory cytokine IL- 6 by enzyme-linked immunosorbent assay (ELISA). DCMAB inhibited 30\% of IL-6 release at $25 \mu \mathrm{g} / \mathrm{mL}$, when compared with controls while HEAB was inactive. $\mathrm{IC}_{50}$ values determined for $\mathbf{2}$ and $\mathbf{3}$ were 3 -fold higher than $\mathbf{1}$. The DCMAB activity seems to be linked to higher proportions of compounds $\mathbf{2}$ and $\mathbf{3}$ in this extract. These observations could thus explain the traditional use of $A$. brachypoda roots in the treatment of osteoarthritis.
\end{abstract}

Keywords: Arrabidaea brachypoda; flavonoids; anti-inflammatory activity; osteoarthritis; high-speed counter current chromatography; mass-spectrometric quantification

\section{Introduction}

Arrabidaea brachypoda (D.C.) is a shrub native to the Brazilian region of Cerrado (neotropical savanna). It belongs to the Bignoniaceae family, which includes 120 genera and nearly 800 species of 
different plants scattered in tropical and subtropical regions worldwide [1]. Plants of Arrabidaea genus are known sources of C-glucosylxanthones, phenylpropanoids, flavonoids, anthocyanidins, allantoins, and triterpenes [2-4]. These molecules are linked to the astringent, anti-inflammatory, antimicrobial, antitumoral and wound healing properties that plants of this genus are known for in traditional medicine [5]. In Brazil, Arrabidaea brachypoda is known as "cervejinha do campo", a decoction of its roots used to treat kidney stones and arthritic joints [6,7]. Different molecules have been isolated and identified from the hydroethanolic extract (HEAB) and dichloromethane extract (DCMAB). From this last extract, three isolated aglycones — brachydin A (1), brachydin B (2), and brachydin C (3) - have been identified. In the HEAB, among the 14 described molecules, these three dimeric flavonoids exist in their glucoronated form [8,9]. Antinociceptive, anti-inflammatory, anti-Trypanosoma cruzi, gastroprotective, antileishmanial, and antimicrobial activities have been recently reported in different in vitro and in vivo assays from both root extracts and isolated compounds [8-15]. In these previous studies, different oral and/or topical administration setups were explored. Overall, studies reporting on anti-inflammatory and antinociceptive activities of $A$. brachypoda have focused on general mechanisms of pain and inflammation [10,12]. In this study, we aimed to test the in vitro anti-inflammatory activity of $A$. brachypoda root extracts and three isolated dimeric flavonoids in the context of osteoarthritis (OA). This chronic disease has worldwide incidence, in particular in the aging population and is considered the most common form of arthritis. Characterized by chronic pain and inflammation, articular cartilage degeneration, and structural changes of whole joints, OA represents the main cause of physical disability and a great health economic burden [16-19]. Presently, treatment options are primarily based on non-pharmacological and symptom management approaches. There is a need for long-acting, targeted local anti-inflammatory drug products, in order to tackle the main OA complications: pain and inflammation [20,21]. Human fibroblast-like synoviocytes activated for OA-like inflammation were used as the in vitro cellular model. Interleukin-6 (IL-6) is a ubiquitous pro-inflammatory cytokine of acute inflammation, particularly involved in the synovial hypertrophy, as well as an established therapeutic target for arthritis. Therefore, IL-6 was selected as the molecular marker of inflammation in this study [21]. Additionally, the isolated compounds were quantified in both HEAB and DCMAB to establish a link between the chemical composition of the extracts and anti-inflammatory properties of their individual constituents.

\section{Results and Discussion}

\subsection{Isolation of Compounds $\mathbf{1}-\mathbf{3}$ from the DCMAB of A. brachypoda}

The DCMAB of A. brachypoda was obtained according to the protocol described in our previous study [8]. The high-performance liquid chromatography with photodiode array detection (HPLC-PDA) analysis revealed three major compounds (Figure 1) which were previously characterized as three unusual dimeric flavonoids named brachydin A (1), brachydin B (2), and brachydin C (3) [8]. To study the anti-inflammatory properties of these molecules, DCMAB was purified at large scale. As a first step, separation conditions were optimized at HPLC-PDA analytical scale (Figure S1, Supplementary Material) and transferred to medium pressure liquid chromatography (MPLC) at a semi-preparative scale, using a gradient transfer method. This yielded $4 \mathrm{~g}$ of brachydin $\mathrm{A}$ (compound $\mathbf{1}$; Figure 1A) in a single step. Brachydin B (2) and brachydin C (3) were obtained in a mixture (4.9 g) (see Section 3.2.3), mostly due to a column overload, and were further purified by high-speed counter current chromatography (HSCCC). The solvent system was determined using the ARIZONA solvent system approach. The separation was carried out successfully as a result of the different $K p$ determined (compounds $\mathbf{2}(K p=1.5)$ and $\mathbf{3}(K p=2.2)$ (see Section 3.2.3). A full recovery of pure compound $\mathbf{2}(1.4 \mathrm{~g})$ and $\mathbf{3}(3.2 \mathrm{~g}$ ) (Figure 1C) resulted, as HSCCC is a support-free liquid-liquid partition chromatography technique [22]. According to the literature, this is the first time this technique has been described for the isolation of pure compounds from A. brachypoda root extracts. 
A

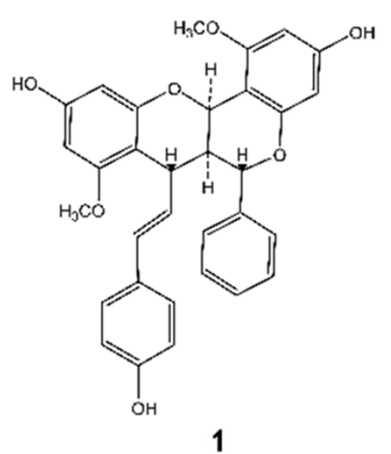

B

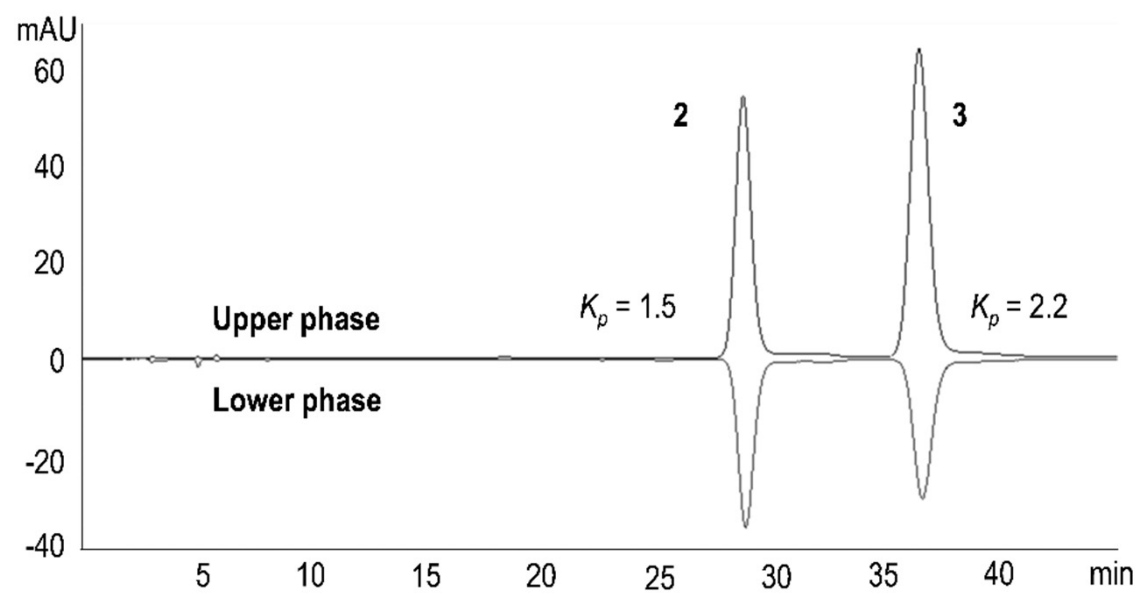

C

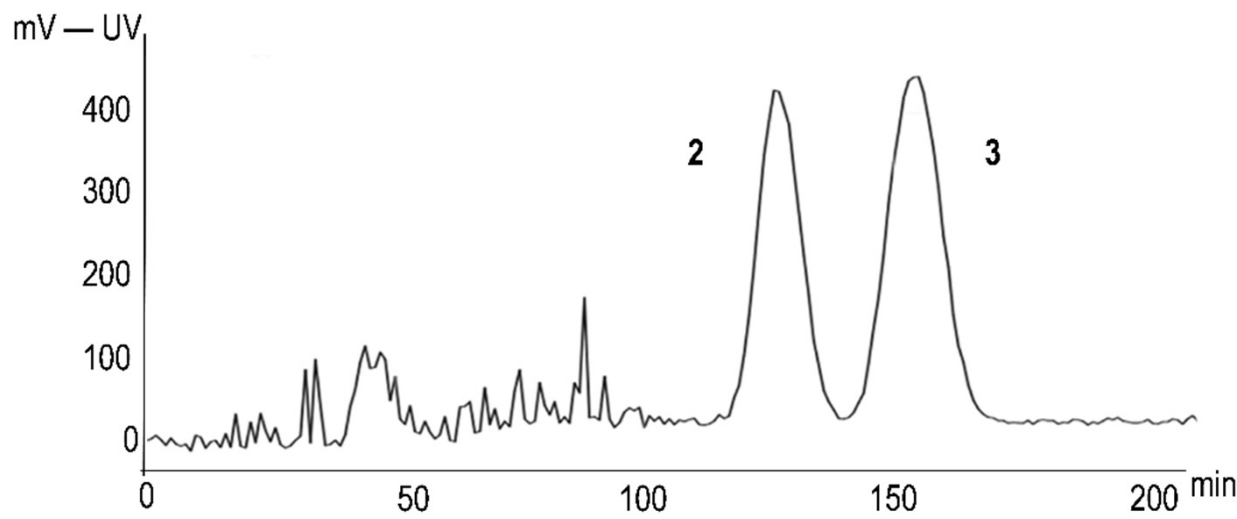

Figure 1. (A) Structures of brachydin A (1), brachydin B (2), and brachydin C (3) isolated from the dichloromethane extract (DCMAB) of $A$. brachypoda roots (B) Determination of coefficient of partition $K p$ by high-performance liquid chromatography with photodiode array detection (HPLC-PDA) of the solvent mixture P: methanol/ethyl acetate/hexane/water $(6: 5: 6: 5 ; v / v / v / v)$ of the ARIZONA system [23]. (C) High-speed counter current chromatography (HSCCC)-UV chromatogram at $280 \mathrm{~nm}$, with clear separation of compounds 2 and 3 . 


\subsection{Quantification of Compounds 1-3 from A. brachypoda Root Extracts by UHPLC-MS/MS}

The amounts of compounds 1, 2, and 3 in the HEAB and DCMAB were determined by ultrahigh pressure liquid chromatography coupled to mass spectrometer (UHPLC-MS/MS) analysis, in the multiple reaction monitoring (MRM) mode. The MRM parameters of each analyte were optimized to increase sensitivity for a specific transition from mass spectrum ${ }^{2}\left(\mathrm{MS}^{2}\right)$ reported data (Table S1, Supplementary Material) [8]. The range of the calibration curves was estimated for each compound and was set to $31-500 \mathrm{ng} / \mathrm{mL}$. This yielded five data point calibration curves with $\mathrm{r}^{2}>0.99$ (Table S2, Supplementary Material). The results obtained from the quantitative analysis are displayed in Figure 2. The total content of compounds 1-3 was 3.35-fold higher in DCMAB than in HEAB. This was expected, since these compounds are primarily occurring as glucoronated derivatives in HEAB [8], and these are not detected by MRM. Compound 3 was the most abundant in both DCMAB (123 mg/g (DW: dry weight) and HEAB (36 mg/g (DW)). Compounds 1 and 2 were present at 16 and $108 \mathrm{mg} / \mathrm{g}(\mathrm{DW})$ in DCMAB, respectively. Lower levels of $1(5 \mathrm{mg} / \mathrm{g})$ and $2(33 \mathrm{mg} / \mathrm{g}(\mathrm{DW}))$ were found in HEAB. These results quantify, for the first time, the amounts of the three isolated brachydins in both extracts.

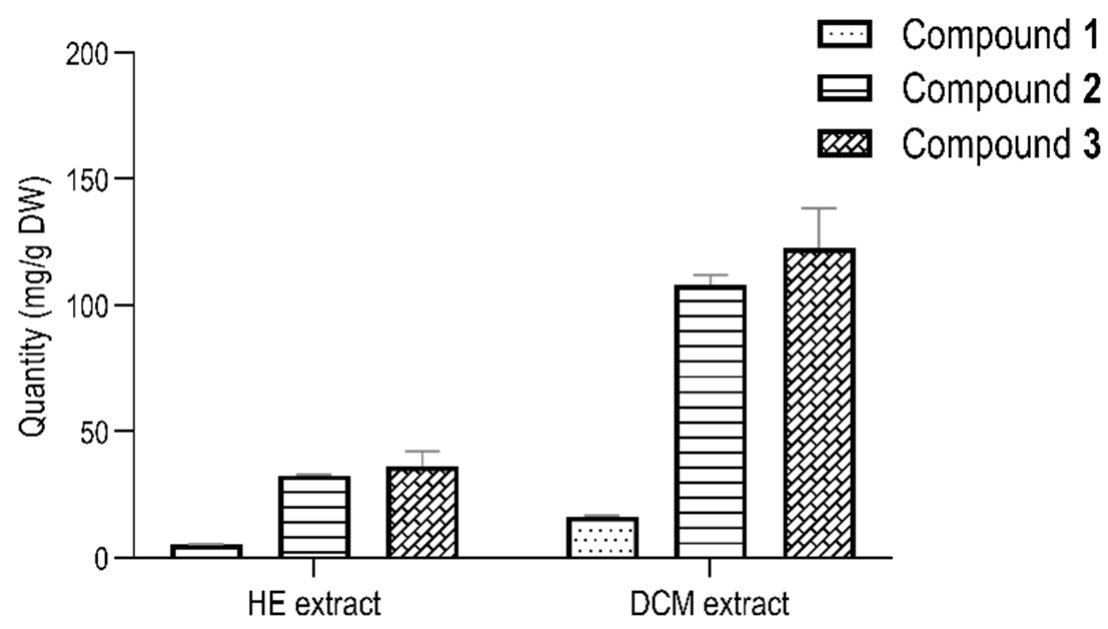

Figure 2. Concentration (mg/g DW) of compounds 1-3 in hydroethanolic extract (HEAB) and dichloromethane extract (DCMAB), respectively. Bars correspond to mean values \pm S.D.; $n=3$.

\subsection{In Vitro Cytotoxicity and Anti-Inflammatory Bioactivity of Extracts and Isolated Compounds}

\subsubsection{Arrabidaea bachypoda Extracts (HEAB and DCMAB)}

The cytotoxicity of both HEAB and DCMAB was assessed; results are shown in Figure 3A (and corresponding scatter plot in Figure S4). Human fibroblast like synoviocytes (HFLS) were viable after $24 \mathrm{~h}$ incubation, with tested concentrations ranging from 3 to $100 \mu \mathrm{g} / \mathrm{mL}$ of HEAB. Conversely, at $50 \mu \mathrm{g} / \mathrm{mL}$ and $100 \mu \mathrm{g} / \mathrm{mL}$ of DCMAB, HFLS presented viability values of $68 \%$ and $5 \%$, respectively, compared to controls (Figure 3A). Consequently, these two top concentrations were not considered in the bioactivity assay, a sandwich enzyme-linked immunosorbent assay (ELISA) (Figure 4A), due to toxicity. At the highest dose tested $(100 \mu \mathrm{g} / \mathrm{mL})$, HEAB showed a $30 \%$ inhibition of IL-6 release. DCMAB yielded a similar inhibitory effect at $25 \mu \mathrm{g} / \mathrm{mL}$ (31\% inhibition; Table 1), the highest concentration inducing no cytotoxicity. Both inhibition effects were non-significant ( $p$ value 0.27 and 0.19 , respectively) when compared to non-treated control: interleukin-1 beta (IL-1 $\beta$ ). In line with these results, $\mathrm{IC}_{50}$ calculations (Table 1 ) show that an anti-inflammatory effect occurs only above $100 \mu \mathrm{g} / \mathrm{mL}$ and $31 \mu \mathrm{g} / \mathrm{mL}$ for $\mathrm{HEAB}$ and $\mathrm{DCMAB}$, respectively. 


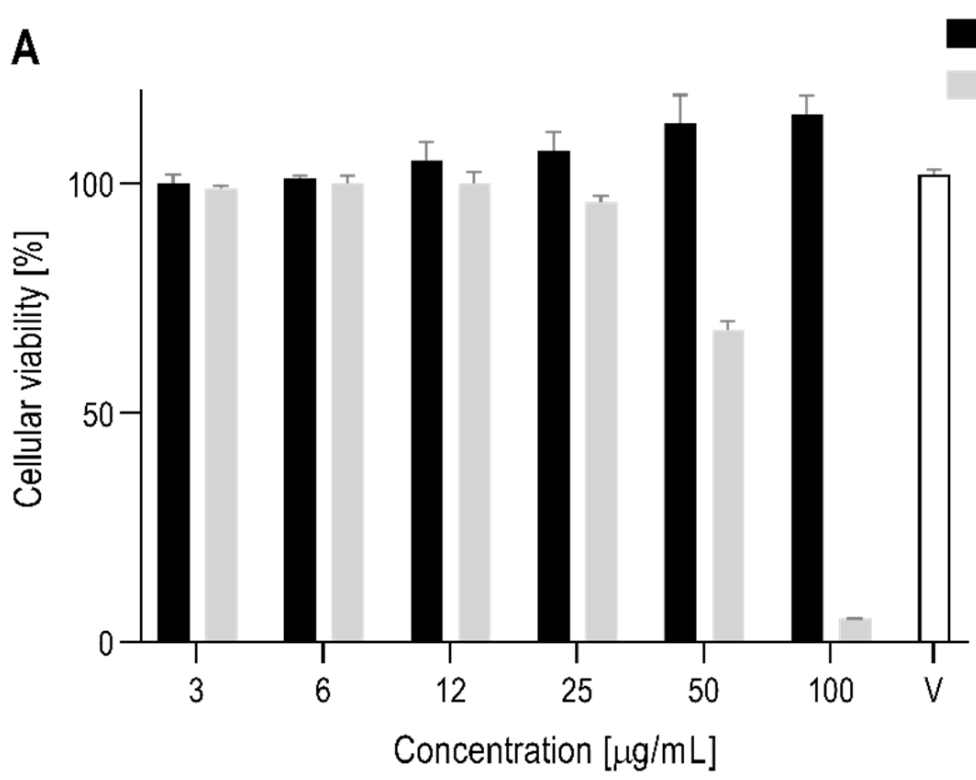

- $\mathrm{HEAB}$

DCMAB

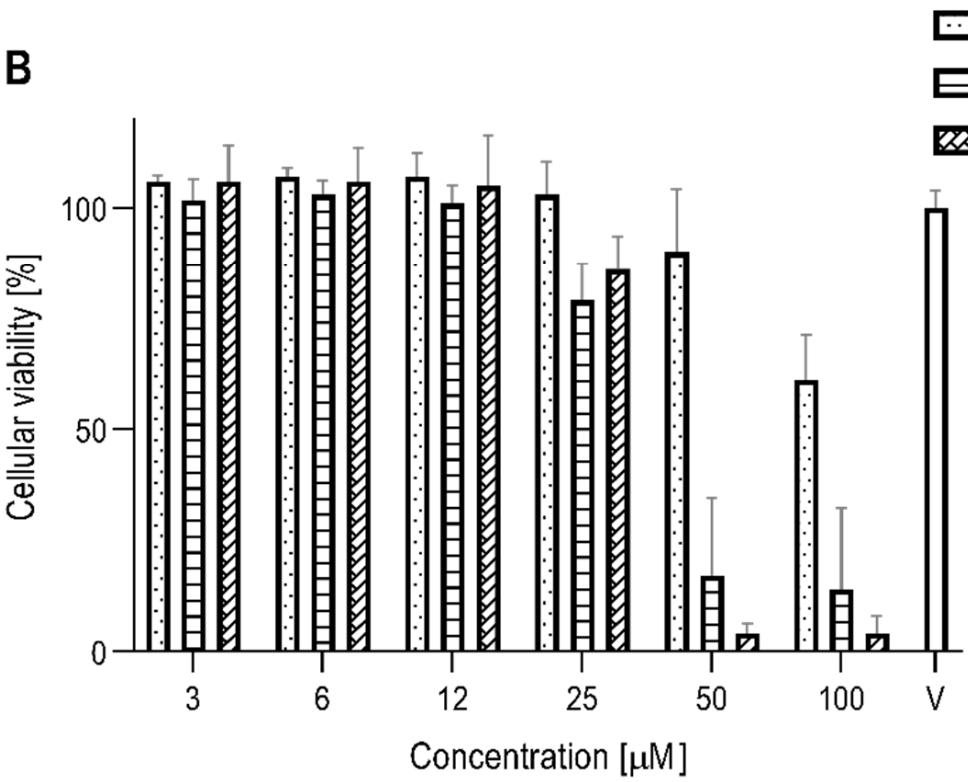

Figure 3. The cellular viability of human fibroblast like synoviocytes (HFLS) incubated with all tested compounds at increasing concentrations, after $24 \mathrm{~h}$. Root extracts (A) and isolated compounds from dichloromethane extract (DCMAB) (B). Bars correspond to mean values \pm S.D.; $n=6$; V = vehicle, $0.01 \%$ dimethyl sulfoxide (DMSO). Additional scatter plot with individual data in Supplementary Material, Figure S4. 


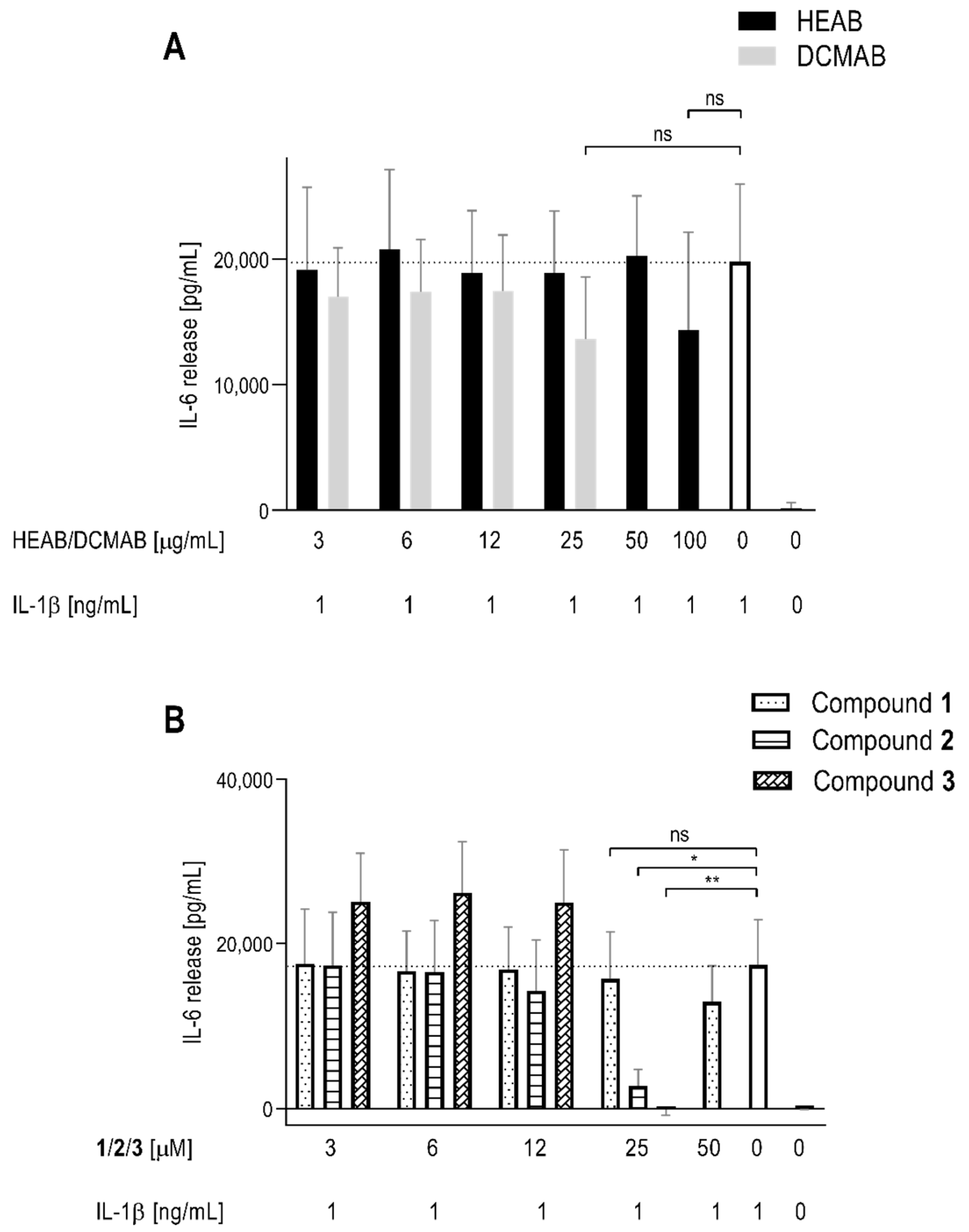

Figure 4. Release of pro-inflammatory cytokine IL- 6 from IL-1 $\beta$ activated HFLS incubated with root extracts (A) and isolated compounds 1-3 (B), after $24 \mathrm{~h}$. Bars correspond to mean values \pm S.D.; $n=6$. ${ }^{*} p<0.05,{ }^{* *} p<0.009$ and $\mathrm{ns}=$ no significance. Dotted lines represent cut-off of maximum IL-6 release by Il- $1 \beta$ stimulation. 
Table 1. IL-6 inhibitory activity and anti-inflammatory $\mathrm{IC}_{50}$ of all tested compounds.

\begin{tabular}{|c|c|c|c|c|c|c|}
\hline Extracts & & \multicolumn{2}{|c|}{$\mathrm{IC}_{50}(\mu \mathrm{g} / \mathrm{mL})$} & \multicolumn{3}{|c|}{$\begin{array}{l}\text { Inhibition of IL-6 Release at Highest non-Toxic } \\
\text { Concentration }(\%)[25 \mu \mathrm{g} / \mathrm{mL}]\end{array}$} \\
\hline HEAB & & \multicolumn{2}{|c|}{$>100$} & \multicolumn{3}{|c|}{$2 \pm 12$} \\
\hline DCMAB & & \multicolumn{2}{|c|}{$31 \pm 1$} & \multicolumn{3}{|c|}{$31 \pm 8$} \\
\hline Compounds & $\begin{array}{l}\text { Molecular } \\
\text { Weight } \\
(\mathrm{g} / \mathrm{mol})\end{array}$ & $(\mu \mathrm{g} / \mathrm{mL})$ & $(\mu \mathrm{M})$ & $\begin{array}{c}\text { Inhibition of IL-6 } \\
\text { release at highest } \\
\text { non-toxic } \\
\text { concentration }(\%) \\
{\left[25 \mu \mathrm{M}=13 \mu \mathrm{g} / \mathrm{mL}^{*}\right]}\end{array}$ & $\begin{array}{l}\text { Compound } \\
\text { in DCMAB } \\
\left(\% \text { of DW }{ }^{* *}\right)\end{array}$ & $\begin{array}{c}\text { Compound } \\
\text { concentration in } \\
\mathrm{IC}_{50} \text { of DCMAB } \\
(\mu \mathrm{g} / \mathrm{mL})\end{array}$ \\
\hline 1 & 524 & $33 \pm 2$ & $62 \pm 2$ & $10 \pm 11$ & 2 & 0.5 \\
\hline 2 & 538 & $9 \pm 3$ & $17 \pm 3$ & $80 \pm 9$ & 10 & 3.4 \\
\hline 3 & 508 & $10 \pm 3$ & $19 \pm 3$ & $94 \pm 5$ & 12 & 3.8 \\
\hline
\end{tabular}

${ }^{*}$ Mean value for equivalence to $25 \mu \mathrm{M}$ of Compound 1,2 and $3 .{ }^{* *}$ Dry Weight (DW).

\subsubsection{Isolated Brachydins from DCMAB (1-3)}

The three isolated compounds 1, 2, and 3, were also tested for cytotoxicity (WST-1) and anti-inflammatory activity (ELISA) and showed different activities (Figures 3B, S4 and 4B). Compound 1 was only cytotoxic at $100 \mu \mathrm{M}$, whereas compounds 2 and 3 induced a loss of viability at $50 \mu \mathrm{M}$, with no evident effect of dose increase, unlike what was previously described for DCMAB in terms of cytotoxicity (Figure 3). For this reason, the IL-6 ELISA assay was performed using supernatants recovered after incubation with HFLS, up to $50 \mu \mathrm{M}$ for $\mathbf{1}$ and only up to $25 \mu \mathrm{M}$ for $\mathbf{2}$ and $\mathbf{3}$ (Figure $4 \mathrm{~B}$ ). At the highest concentration with remaining favorable viability $(25 \mu \mathrm{M})$, both 2 and 3 significantly decreased IL-6 release when compared to the activation control IL-1 $\beta$ ( $80 \%$ and $94 \%$ inhibition, respectively (Table 1). Compound $\mathbf{1}$ showed a non-significant inhibition of IL-6 release at $25 \mu \mathrm{M}$ and $50 \mu \mathrm{M}(10 \%$ and $24 \%$, respectively; Figures $4 \mathrm{~B}$ and S5, Supplementary Material). In Table 1, IC 50 values (individual plots represented in Supplementary Material, Figure S6) confirmed the increased anti-inflammatory effect of $\mathbf{2}(17 \mu \mathrm{M})$ and $3(19 \mu \mathrm{M})$, in comparison to $\mathbf{1}(62 \mu \mathrm{M})$. These different outcomes between compounds could be linked to the fact that both compounds 2 and 3 are of higher structural similarity between each other than compound 1, that exhibits higher polarity influencing effects. Regarding cell viability of HFLS, HEAB and compound $\mathbf{1}$ presented the least cytotoxicity and DCMAB, compounds $\mathbf{2}$ and $\mathbf{3}$, showed higher cytotoxicity. This is expected, since these are the major two components of this extract, as above mentioned (see Section 2.2). In order to compare the bioactivity results between the tested compounds, $\mathrm{IC}_{50}$ results in $\mu \mathrm{M}$ (isolated compounds) were transformed into $\mu \mathrm{g} / \mathrm{mL}$ to match those of the extracts, that represent mixtures of several compounds (Table 1 ). In terms of the anti-inflammatory effect, results are in line with those of cytotoxicity. HEAB (IC $50>100 \mu \mathrm{g} / \mathrm{mL}$ ) was inactive in decreasing release of pro-inflammatory cytokine IL-6 in HFLS. According to what was previously reported for HEAB [9-11], this extract is comprised by the dimeric flavonoid aglycones (1-3) and their various glucoronated derivatives [9]. The glucoronated derivatives appear in higher amount than the corresponding aglycones. In an HFLS in vitro setting, the metabolic pathways that hydrolyze the glucoronated forms into simple aglycones (as it would in the acidic $\mathrm{pH}$ of the stomach) do not occur and the compounds are absorbed as is [24]. Based on such considerations, the lack of activity of HEAB can be thus correlated to the low amounts of $\mathbf{1}, \mathbf{2}$, and $\mathbf{3}$ (Figure 2) and the glucoronated derivatives themselves, inactive in this experimental setup. This is also the reasoning as to why all current studies and evaluations of this extract in vivo occur after per os administration and not local [8-10,12]. DCMAB on the other hand, only contains the three isolated brachydins A, B, and C in their non-glucoronated forms. Such extract is thus more interesting to explore in this experimental setup and when seeking local administration of anti-inflammatory treatments for OA. Albeit through unknown pathways, this study is the first analyzing the anti-inflammatory activity in the specific context of OA. Additionally, it compares effects and quantifies the presence of isolated compounds in both A. brachypoda extracts in order to assess their potential as OA therapeutic alternatives. Compound $\mathbf{1}$ exhibited similar 
anti-inflammatory activity $\left(\mathrm{IC}_{50}\right)$ when compared to DCMAB $(33 \mu \mathrm{g} / \mathrm{mL}$ and $31 \mu \mathrm{g} / \mathrm{mL}$, respectively), whereas compounds $2(9 \mu \mathrm{g} / \mathrm{mL})$ and $3(10 \mu \mathrm{g} / \mathrm{mL})$ showed a 3-fold higher activity. This suggests that the extracts activity results mainly from the presence of the two latter compounds. Taking into account the amounts of each compound 1-3 in the DCMAB (Table 1), the cumulative $\mathrm{IC}_{50}$ of each individual isolated compound $(27 \mu \mathrm{g} / \mathrm{mL})$ does not greatly differ from DCMAB alone $-31 \mu \mathrm{g} / \mathrm{mL}$. Based on these results, no investigation on synergistic effect was pursued. Differences between the anti-inflammatory activities between the three compounds potentially stem from the higher polarity of compound 1, in comparison to 2 and 3.

\section{Materials and Methods}

\subsection{Materials}

\subsubsection{General Experimental Procedures}

All analytical HPLC-PDA analyses were performed using an Agilent Technologies 1260 Infinity system equipped with a photodiode array detector (Agilent Technologies, Santa Clara, CA, USA). Preparative medium pressure liquid chromatography (MPLC) was performed using a system equipped with a C-605 module pump, C-640 UV detector, and C-684 fraction collector all from Büchi (Flawil, Switzerland). The system was controlled by the Sepacore Control software (Büchi AG, Flawil, Switzerland). A coil connected to a resistance was used to control the MPLC column temperature. The column $\left(460 \mathrm{~mm} \times 49 \mathrm{~mm}\right.$ i.d.) was packed with ZEOprep ${ }^{\circledR} \mathrm{C} 18$ as the stationary phase (ZEOprep ${ }^{\circledR}$ C18, 15-25 $\mu \mathrm{m}$; Zeochem, Uetikon am See, Switzerland). Nuclear magnetic resonance (NMR) spectroscopic data were recorded on a Bruker Avance III HD 600 MHz NMR spectrometer equipped with a QCI $5 \mathrm{~mm}$ Cryoprobe and a SampleJet automated sample changer (Bruker BioSpin, Rheinstetten, Germany). Chemical shifts were reported in parts per million $(\delta)$ using the $\mathrm{CD}_{3} \mathrm{OD}$ residual signal $(\delta \mathrm{H} 3 ; \delta \mathrm{C} 49)$ as internal standards for $1 \mathrm{H}$ and $13 \mathrm{C}$ NMR and coupling constants (J) were reported in hertz. High-speed counter-current chromatography (HSCCC) coupled to UV was performed on a Tauto TBE-300B instrument (Tauto Biotech, Shangai, China) equipped with two LC10AD HPLC pumps (Shimadzu, Kyoto, Japan), a 20 mL injection loop, a Knauer K 2501 UV detector (Berlin, Germany) and a C-684 fraction collector from Büchi (Flawil, Switzerland). Solvents used for extraction (methanol, ethyl acetate, hexane) were all of analytical grade. Solvents used in the quantification of the isolated brachydins: methanol, formic acid, acetonitrile and water were of LC-MS grade. All solvents were purchased from Sigma-Aldrich (St. Louis, MO, USA). Milli-Q ${ }^{\circledR}$ water from Merck (Burlington, MA, USA) was used throughout this study. All other chemical products were obtained from Sigma-Aldrich (St. Louis, MO, USA). For the in vitro anti-inflammatory assays, all media solutions and ELISA kits were purchased from Invitrogen (Carlsbad, CA, USA). Fetal bovine serum was purchased from Eurobio (Les Ulis, France). Interleukin 1 beta (IL-1 $\beta$ ) was obtained from R\&D Systems (Bio-Techne, Abingdon, UK).

\subsubsection{Plant Material}

Roots of Arrabidaea brachypoda were collected in April 2010 from the Sant'Ana da Serra farm in João Pinheiro, Minas Gerais, Brazil. The plant was identified at the ICEB of José Badine Herbarium of the Federal University of Ouro Preto by Prof. Maria Cristina Teixeira Braga Messias. A voucher specimen (\#17935) was deposited at the Herbarium of the Federal University of Ouro Preto, Brazil. The plant was collected in accordance with Brazilian authorities (SISGEN \#A451DE4).

\subsection{Extraction and Isolation of Brachydins $A(\mathbf{1}), B(2)$ and $C$ (3)}

\subsubsection{Plant Extraction}

Both HEAB and DCMAB of the roots of Arrabidaea brachypoda were obtained from our previous study [9]. These extracts were stored and protected from light at $-20{ }^{\circ} \mathrm{C}$. 


\subsubsection{HPLC-PDA Analysis}

The DCMAB of A. brachypoda (Figure S1, Supplementary Material), the MPLC and HSCCC fractions were analyzed by HPLC-PDA using a Waters X-Bridge C18 column $(250 \mathrm{~mm} \times 4.6 \mathrm{~mm}$ i.d., $5 \mu \mathrm{m}$; Waters, Milford, MA, USA) equipped with a Waters C18 pre-column cartridge holder $(10 \mathrm{~mm} \times 2.1 \mathrm{~mm}$ i.d.). Water (A) and methanol (B), both containing $0.1 \%$ of formic acid (FA) were used as the solvent system. The column was equilibrated with $5 \%$ of $B$ for $15 \mathrm{~min}$. The separation was performed in gradient mode, as follows: 5 to $100 \%$ of B in $60 \mathrm{~min}$, and $100 \%$ of B for $10 \mathrm{~min}$. Flow rate $1 \mathrm{~mL} / \mathrm{min}$; injection volume $10 \mu \mathrm{L}$; sample concentration $10 \mathrm{mg} / \mathrm{mL}$ in methanol. The UV absorbance was measured at $254 \mathrm{~nm}$ and the UV-PDA spectra were recorded between 190 and $600 \mathrm{~nm}$ (step $2 \mathrm{~nm}$ ).

\subsubsection{Isolation of Brachydins A (1), B (2), and C (3)}

DCMAB was fractioned by MPLC, following a previously published protocol [25]. Fractionation conditions were optimized on an HPLC column (see Sections 3.1.1 and 3.2.2) using the same stationary phase and an acidic $(0.1 \% \mathrm{FA})$ water (A) and methanol (B) gradient. Solvent system gradient went as follows: isocratic $5 \% \mathrm{~B}$ in $56 \mathrm{~min}, 5$ to $38 \% \mathrm{~B}$ in $5.5 \mathrm{~h}$, isocratic $38 \% \mathrm{~B}$ in $4.7 \mathrm{~h}, 38$ to $62 \% \mathrm{~B}$ in $3.2 \mathrm{~h}, 62$ to $100 \% \mathrm{~B}$ in $9 \mathrm{~min}$ and isocratic $100 \% \mathrm{~B}$ during $1.5 \mathrm{~h}$. A dry load injection of the sample was performed by mixing $7 \mathrm{~g}$ of the dichloromethane extract with $35 \mathrm{~g}$ of Zeoprep ${ }^{\circledR} \mathrm{C} 18(40-63 \mu \mathrm{m})$. The dry-load cell $(11.5 \mathrm{~cm} \times 2.7 \mathrm{~cm}$ i.d.) was subsequently connected between the pumps and the MPLC column. The flow rate was set to $20 \mathrm{~mL} / \mathrm{min}$ and UV absorbance was monitored at $280 \mathrm{~nm}$. MPLC separation yielded 90 fractions of $250 \mathrm{~mL}$, which were analyzed by ultrahigh pressure liquid chromatography coupled to an UV detector (UHPLC-UV). This approach yielded gram amounts of compound $\mathbf{1}$. Fractions containing pure compounds were combined, dried, analyzed by nuclear magnetic resonance (NMR) and properly stored. Other MPLC fractions containing mixtures of compounds $\mathbf{2}$ and $\mathbf{3}$ were combined and subjected to purification by HSCCC coupled to a UV detector. The ARIZONA solvent system approach was applied to perform the separation of compounds 2 and 3 . This approach is based on the use of 23 solvent mixture compositions of methanol/ethyl acetate/hexane/water. The mixtures are labeled with letters from the alphabet from A to Z (except E, I, and O) [23]. To determine the best-suited ARIZONA solvent mixture, the partition coefficient $(K p)$ of each compound was determined. As so, few milligrams of the fraction containing $\mathbf{2}$ and $\mathbf{3}$ were solubilized in a specific mix of the ARIZONA system. Upper and lower phases of each mixture of solvents were separated and analyzed by HPLC-UV. The UV area peak of each compound was used to determine its coefficient of partition $(K p)$ according to the equation in Figure S2 (Supplementary Material). The best results for complete separation of compounds 2 and 3 were obtained by solvent mixture P-methanol/ethyl acetate/hexane/water (6:5:6:5; $v / v / v / v)$. The coil was first filled with the two phases (upper and lower, 1:1) and rotation was set to $1000 \mathrm{rpm}$. Lower phase was then pumped into the column at a flow rate of $3 \mathrm{~mL} / \mathrm{min}$ using the head-to-tail mode (mobile phase $=$ lower phase; stationary phase $=$ upper phase) with rotation set at $8000 \mathrm{rpm}$. After equilibrium between the two phases, $500 \mathrm{mg}$ of sample in $20 \mathrm{~mL}$ of upper and lower phase solution (1:1) were injected. Four injections were performed. A total of 35 fractions ( $5 \mathrm{ml}$ each) were obtained for each injection. The fractions were combined according to the chemical composition determined by HPLC-PDA analysis, dried, analyzed by NMR, and properly stored.

\subsection{Quantification of Compounds 1-3 from A. brachypoda Root Extracts by UHPLC-MS/MS}

Sample preparation was carried out by dissolving the dried HEAB and DCMAB from Arrabidaea brachypoda roots in $100 \%$ methanol. The final concentration of the samples was $10 \mu \mathrm{g} / \mathrm{mL}$, and three technical replicates were used for quantitative analysis. The amounts of compounds $\mathbf{1}, \mathbf{2}$, and 3 in the extracts were quantified on a QTRAP 4000 quadrupole linear ion-trap mass spectrometer (Sciex, Darmstadt, Germany) with an ESI interface operating in positive ionization mode. UHPLC was performed on an Acquity UPLC system (Waters, Milford, MA, USA) equipped with a Waters UPLC BEH C18 column $(50 \mathrm{~mm} \times 2.1 \mathrm{~mm}$ ID, $1.7 \mu \mathrm{m})$. The solvent system consisted of water: $0.1 \%$ FA for 
solvent A and acetonitrile: 0.1\% FA for solvent B. The elution was performed in gradient mode at $40{ }^{\circ} \mathrm{C}(400 \mu \mathrm{L} / \mathrm{min}$ flow rate) with the following steps: 5 to $60 \% \mathrm{~B}$ in $4.2 \mathrm{~min}, 60$ to $70 \% \mathrm{~B}$ in $1.3 \mathrm{~min}$, 70 to $100 \%$ B in $1.5 \mathrm{~min}$, maintaining B at $100 \%$ for $1 \mathrm{~min}$ and lastly, a reconditioning with $5 \% \mathrm{~B}$ for min. The autosampler compartment was set at $10{ }^{\circ} \mathrm{C}$ throughout the analysis and the injection volume was $5 \mu \mathrm{L}$. MS/MS quantitative analyses were performed in multiple reaction monitoring (MRM) mode. Source and gas parameters were as follows: curtain gas, 10 psi; collision gas, 10 psi; ionspray voltage, $3500 \mathrm{~V}$; source temperature, $40{ }^{\circ} \mathrm{C}$; ion source gas $1,40 \mathrm{psi}$; ion source gas $2,50 \mathrm{psi}$. Nitrogen was used as collision gas. MRM transitions and MRM parameters were all optimized via flow injection analysis (FIA) technique with a mix containing $10 \mathrm{ng} / \mathrm{mL}$ of pure $\mathbf{1}, \mathbf{2}$, and $\mathbf{3}$ analytes (Table S1, Supplementary Material). Quantification was achieved by constructing three separate external calibration curves from solutions of the isolated pure compounds. The calibration curve concentrations for active compounds were as follows: $500 \mathrm{ng} / \mathrm{mL}, 250 \mathrm{ng} / \mathrm{mL}, 125 \mathrm{ng} / \mathrm{mL}, 63 \mathrm{ng} / \mathrm{mL}$, $31 \mathrm{ng} / \mathrm{mL}$. As no analyte-free matrix was available, the displayed limits of quantification (LOQ) were evaluated only on each pure standard. A signal-to-noise ratio of 10 was considered as the approximate LOQ (Table S2, Supplementary Material).

\subsection{In Vitro Anti-Inflammatory Bioactivity}

\subsubsection{Human Fibroblast-like Synoviocytes (HFLS) Isolation and Culture}

Hip synovial membrane was collected from three male adult patients with clinical osteoarthritis $(\mathrm{OA})$ at the time of hip replacement surgery. This protocol was conducted under the approval of the local Ethics Committee (CCER, Geneva, Switzerland) (Authorization \#2017-02234) and with informed and consenting patients. Once collected, tissue samples were processed, according to previously described protocols [26]. Briefly, samples were finely minced and digested for $3 \mathrm{~h}\left(37^{\circ} \mathrm{C}, 5 \% \mathrm{CO}_{2}\right.$ incubation) in a $3 \mathrm{mg} / \mathrm{mL}$ collagenase IX-RPMI 1640 solution. After centrifugation $(200 \times g)$ and supernatant removal, the resuspended pellet was cultured $\left(37^{\circ} \mathrm{C}, 5 \% \mathrm{CO}_{2}\right)$ in medium containing RPMI 1640, M199 (1:1), 1\% penicillin/streptomycin (100 IU/mL:100 g/mL), 2 mM L-glutamine and $20 \%$ fetal bovine serum. After overnight culture, non-adherent cells were removed. At confluence, cells were trypsinized and passaged to $75 \mathrm{~cm}^{2}$ culture flasks (Corning, NY, USA) in complete medium containing $10 \%$ fetal bovine serum. A double control was performed to confirm presence of HFLS after isolation: visual morphology evaluation (Figure S3) and flow cytometry with synoviocyte specific surface markers (CD14 ${ }^{+}$and VCAM-1). A representative sample of all cell populations was found to have above $90 \%$ HFLS markers. All cellular assays were performed from passage 3 to 9 . Experiments were conducted twice per donor $(n=6)$.

\subsubsection{In Vitro Cytotoxicity Assay and Bioactivity Assay with the Determination of IL-6 Release in HFLS}

Confluent HFLS cells (25,000 cells/well) were treated in a 96-well plate with compounds 1, 2, and $3(3 \mu \mathrm{M}, 6 \mu \mathrm{M}, 12 \mu \mathrm{M}, 25 \mu \mathrm{M}, 50 \mu \mathrm{M}$, and $100 \mu \mathrm{M})$, HEAB and DCMAB (increasing concentrations from 3 to $100 \mu \mathrm{g} / \mathrm{mL}$ ), or control vehicle for $1 \mathrm{~h}$. All tested compounds were dissolved in a dimethyl sulfoxide (DMSO) stock solution and further diluted in culture medium. Final concentration of DMSO in vehicle of tested concentrations was $0.01 \%$. After $1 \mathrm{~h}$ incubation, cells were activated by addition of IL-1 $\beta(1 \mu \mathrm{g} / \mathrm{mL})($ R\&D Systems, Bio-Techne, Abingdon, UK) with subsequent incubation for $23 \mathrm{~h}$. All supernatants were kept at $-80^{\circ} \mathrm{C}$, before further testing. Remaining adherent cells were tested for viability using the cell proliferation reagent WST-1 (Roche, Basel, Switzerland), according to the supplier instructions. Pro-inflammatory cytokine IL-6 release in cell supernatant was assessed by ELISA. For this, a human IL-6 ELISA kit from Invitrogen (ref \#88-7066-88; Thermo-Fisher Scientific, Waltham, MA, USA) was used according to the manufacturer's protocol. All samples were diluted 90 times. Experiments were conducted twice per donor $(n=6)$. 


\subsection{Half Maximal Inhibitory Concentration $\left(I_{50}\right)$ Determination and Statistical Analysis}

In this study, data are presented as mean values \pm standard deviation (S.D.). All analyses were performed using GraphPad Prism 8.3 software. IC $_{50}$ values were determined using a nonlinear fitting of a $\log$ (inhibitor concentration) vs response variable slope for each donor's data set. Consequent values were then averaged. After confirming the data normal distribution and homogeneity of variances (Brown-Forsythe and Welch; Shapiro-Wilk, D'Agostino-Pearson and Anderson-Darling), statistical analysis was performed using a two-way ANOVA with a Bonferroni multiple comparisons test. Significance was determined at alpha level 0.05. Significance values represented are ${ }^{*} p<0.05$, ** $p<0.009$ and ns for non-significance.

\section{Conclusions}

Arrabidaea brachypoda is a plant commonly used in Brazilian traditional medicine to treat pain and inflammation related to arthritic joints. The effects of HEAB, DCMAB and three isolated dimeric flavonoids were assessed. In this study, specific in vitro anti-inflammatory activity was tested using primary human arthritic synoviocytes, in order to understand the mechanisms involved and potentially identify molecules of interest for local drug delivery in OA. The present study is the first comparing the anti-inflammatory effect and providing a quantification of the three isolated compounds from the DCMAB. Despite high cellular viability scores at tested concentrations, HEAB was not performant in terms of anti-inflammatory effect. Studies suggest that the acidic hydrolysis in the stomach is responsible for the releasing of aglycones deriving from glucoronated forms of not only brachydins $\mathrm{A}$, $B$, and $C$, but also other molecules of the extract [8,24]. In this study setup however, hydrolysis does not take place, which explains the lack of activity of HEAB. Conversely, DCMAB showed an effect against the release of pro-inflammatory cytokine IL-6. Quantification of 1, 2, and 3 in this extract showed higher proportions of 2 and 3 compared to 1 . The bioactivity results are in line with these findings, where $\mathbf{1}$ alone is considerably less active than to $\mathbf{2}$ and $\mathbf{3}$ alone. Compound $\mathbf{3}$ presented as both the most effective and cytotoxic compound, the fact that it is the main component of DCMAB could account for the extract's enhanced effect over both $\mathbf{1}$ and $\mathbf{2}$ and, partly, HEAB. Differences in polarity between compound 1 versus 2 and 3 can affect bioavailability and bioactivity. Compounds 2 and 3 seem to have a major role in the anti-inflammatory effects of DCMAB and, potentially, other aglycones present in HEAB deserve further exploring in terms of anti-inflammatory bioactivity. Additionally, further research of in vivo studies and potential drug delivery systems would enrich the understanding of the mechanisms underlying A. brachypoda roots and its treatment of arthritic joints.

Supplementary Materials: The following are available online, Figure S1: Reverse phase HPLC-UV analysis at $254 \mathrm{~nm}$, Figure S2: Coefficient of partition Kp equation, Figure S3: Human fibroblast like synoviocytes, Figure S4: Plot of individual values of cellular viability of extracts and isolated compounds, Figure S5: Inhibition percentage of IL-6 release normalized to $100 \%$ activation, Figure S6: IC $_{50}$ calculations of extracts and isolated compounds, Table S1: Optimized MRM parameters for the quantification of active compounds by UHPLC-MS/MS, Table S2: Calibration curves parameters for active compounds and determination of the LOD and LOQ in $\mathrm{ng} / \mathrm{mL}$.

Author Contributions: Conceptualization, E.F.Q., O.J., E.A.; methodology, C.S., H.M., N.C.d.A., L.N., L.M., C.Q.d.R., W.V., E.F.Q., O.J., E.A.; validation, L.N., L.M., E.F.Q., O.J., E.A.; formal analysis, C.S., H.M., L.N., L.M., O.J.; investigation, C.S., H.M., N.C.d.A., L.M.; resources, E.F.Q., J.-L.W., O.J., E.A.; data curation, C.S., H.M., N.C.d.A., L.N., L.M., E.F.Q., O.J., E.A.; writing-original draft preparation, C.S., H.M., L.M., E.F.Q., O.J., E.A.; writing-review and editing, L.M., E.F.Q., O.J., E.A., J.-L.W.; visualization, C.S., O.J., E.A., E.F.Q., L.M.; supervision, E.F.Q., J.-L.W., O.J., E.A.; project administration, E.F.Q., E.A.; funding acquisition, E.F.Q., J.-L.W., O.J., E.A. All authors have read and agreed to the published version of the manuscript.

Funding: This research was funded by the University of Geneva.

Acknowledgments: The authors would like to thank Thanise Füller for her help in the isolation of compounds 1-3.

Conflicts of Interest: The authors declare no conflict of interest. 


\section{References}

1. Von Poser, G.L.; Schripsema, J.; Henriques, A.T.; Jensen, S.K. The distribution of iridoids in Bignoniaceae. Biochem. Syst. Ecol. 2000, 28, 351-366. [CrossRef]

2. Pauletti, P.M.; Castro-Gamboa, I.; Silva, D.H.S.; Young, M.C.M.; Tomazela, D.M.; Eberlin, M.N.; Bolzani, V.S. New AntioxidantC-Glucosylxanthones from the Stems ofArrabidaea samydoides. J. Nat. Prod. 2003, 66, 1384-1387. [CrossRef]

3. González, B.; Suarez-Roca, H.; Bravo, A.; Salas-Auvert, R.; Avila, D. Chemical Composition and Biological Activity of Extracts from Arrabidaea Bilabiata. Pharm. Biol. 2000, 38, 287-290. [CrossRef]

4. Martin, F.; Hay, A.-E.; Cressend, D.; Reist, M.; Vivas, L.; Gupta, M.P.; Carrupt, P.-A.; Hostettmann, K. AntioxidantC-Glucosylxanthones from the Leaves ofArrabidaea patellifera. J. Nat. Prod. 2008, 71, 1887-1890. [CrossRef]

5. Leite, J.P.V.; De Oliveira, A.B.; Lombardi, J.A.; Filho, J.D.S.; Chiari, E. Trypanocidal activity of triterpenes from Arrabidaea triplinervia and derivatives. Biol. Pharm. Bull. 2006, 29, 2307-2309. [CrossRef]

6. Alcerito, T.; Barbo, F.E.; Negri, G.; Santos, D.; Méda, C.I.; Young, M.C.M.; Chávez, D.; Blatt, C.T. Foliar epicuticular wax of Arrabidaea brachypoda: Flavonoids and antifungal activity. Biochem. Syst. Ecol. 2002, 30, 677-683. [CrossRef]

7. Rodrigues, E.; Mendes, F.; Negri, G. Plants Indicated by Brazilian Indians for Disturbances of the Central Nervous System: A Bibliographical Survey. Central Nerv. Syst. Agents Med. Chem. 2006, 6, 211-244. [CrossRef]

8. Da Rocha, C.Q.; Queiroz, E.F.; Meira, C.S.; Moreira, D.R.M.; Soares, M.B.P.; Marcourt, L.; Vilegas, W.; Wolfender, J.-L. Dimeric Flavonoids from Arrabidaea brachypoda and Assessment of Their Anti-Trypanosoma cruzi Activity. J. Nat. Prod. 2014, 77, 1345-1350. [CrossRef]

9. Da Rocha, C.Q.; De-Faria, F.M.; Marcourt, L.; Ebrahimi, S.N.; Kitano, B.T.; Ghilardi, A.F.; Ferreira, A.L.; Almeida, A.C.A.; Dunder, R.J.; Souza-Brito, A.R.M.; et al. Gastroprotective effects of hydroethanolic root extract of Arrabidaea brachypoda: Evidences of cytoprotection and isolation of unusual glycosylated polyphenols. Phytochem. 2017, 135, 93-105. [CrossRef] [PubMed]

10. Da Rocha, C.Q.; Vilela, F.C.; Cavalcante, G.P.; Santa-Cecília, F.V.; Santos-E-Silva, L.; Dos Santos, M.H.; Giusti-Paiva, A. Anti-inflammatory and antinociceptive effects of Arrabidaea brachypoda (DC.) Bureau roots. J. Ethnopharmacol. 2011, 133, 396-401. [CrossRef]

11. Resende, F.A.; Nogueira, C.H.; Espanha, L.G.; Boldrin, P.K.; Oliveira-Höhne, A.P.; De Camargo, M.S.; Da Rocha, C.Q.; Vilegas, W.; Varanda, E.A. In vitro toxicological assessment of Arrabidaea brachypoda (DC.) Bureau: Mutagenicity and estrogenicity studies. Regul. Toxicol. Pharmacol. 2017, 90, 29-35. [CrossRef] [PubMed]

12. Rodrigues, V.P.; Da Rocha, C.Q.; Périco, L.L.; Santos, R.D.C.D.; Ohara, R.; Nishijima, C.M.; Queiroz, E.F.; Wolfender, J.-L.; Da Rocha, L.R.M.; Santos, A.R.S.; et al. Involvement of Opioid System, TRPM8, and ASIC Receptors in Antinociceptive Effect of Arrabidaea brachypoda (DC) Bureau. Int. J. Mol. Sci. 2017, 18, 2304. [CrossRef] [PubMed]

13. Rocha, V.P.C.; Da Rocha, C.Q.; Queiroz, E.F.; Marcourt, L.; Vilegas, W.; Grimaldi, G.B.; Furrer, P.; Allémann, E.; Wolfender, J.-L.; Soares, M.B.P. Antileishmanial Activity of Dimeric Flavonoids Isolated from Arrabidaea brachypoda. Molecules 2018, 24, 1. [CrossRef]

14. Andrade, L.M.D.S.; De Oliveira, A.B.M.; Leal, A.L.A.B.; Oliveira, F.A.D.A.; Portela, A.L.; Neto, J.D.S.L.; De Siqueira-Júnior, J.P.; Kaatz, G.W.; Da Rocha, C.Q.; Barreto, H.M. Antimicrobial activity and inhibition of the NorA efflux pump of Staphylococcus aureus by extract and isolated compounds from Arrabidaea brachypoda. Microb. Pathog. 2020, 140, 103935. [CrossRef] [PubMed]

15. Da Rocha, C.Q.; Vilela, F.C.; Santa-Cecília, F.V.; Cavalcante, G.P.; Vilegas, W.; Giusti-Paiva, A.; Santos, M.H. Oleanane-type triterpenoid: An anti-inflammatory compound of the roots Arrabidaea brachypoda. Rev. Bras. Farm. 2015, 25, 228-232. [CrossRef]

16. Goldring, M.B.; Goldring, S.R. Osteoarthritis. J. Cell. Physiol. 2007, 213, 626-634. [CrossRef] [PubMed]

17. Nelson, A.E.; Allen, K.D.; Golightly, Y.M.; Goode, A.P.; Jordan, J.M. A systematic review of recommendations and guidelines for the management of osteoarthritis: The Chronic Osteoarthritis Management Initiative of the U.S. Bone and Joint Initiative. Semin. Arthritis Rheum. 2014, 43, 701-712. [CrossRef] 
18. Koszowska, A.; Hawranek, R.; Nowak, J. Osteoarthritis-A multifactorial issue. Reumatologia 2014, 5, 319-325. [CrossRef]

19. Hunter, D.J.; Schofield, D.J.; Callander, E.J. The individual and socioeconomic impact of osteoarthritis. Nat. Rev. Rheumatol. 2014, 10, 437-441. [CrossRef]

20. Kolasinski, S.L.; Neogi, T.; Hochberg, M.C.; Oatis, C.; Guyatt, G.; Block, J.; Callahan, L.; Copenhaver, C.; Dodge, C.; Felson, D.; et al. 2019 American College of Rheumatology/Arthritis Foundation Guideline for the Management of Osteoarthritis of the Hand, Hip, and Knee. Arthritis Rheumatol. 2020, 72, 220-233. [CrossRef]

21. Abramoff, B.; Caldera, F.E. Osteoarthritis. Med Clin. North Am. 2020, 104, 293-311. [CrossRef] [PubMed]

22. Hostettmann, P.D.K.; Hostettmann, M.; Marston, A. Counter-Current Chromatography. In Preparative Chromatography Techniques; Springer Science and Business Media LLC: Berlin, Germany, 1986; pp. 80-126.

23. Foucault, A.P. Centrifugal Partition Chromatography; Chromatographic Science Series; CRC Press: Cleveland, OH, USA, 1995.

24. Hill, R.M.; Lewis, H.B. The Hydrolysis of Sucrose in the Human Stomach. Am. J. Physiol. Content 1922, 59, 413-420. [CrossRef]

25. Challal, S.; Queiroz, E.F.; Debrus, B.; Kloeti, W.; Guillarme, D.; Gupta, M.P.; Wolfender, J.-L. Rational and Efficient Preparative Isolation of Natural Products by MPLC-UV-ELSD based on HPLC to MPLC Gradient Transfer. Planta Medica 2015, 81, 1636-1643. [CrossRef]

26. Neff, L.; Zeisel, M.B.; Druet, V.; Takeda, K.; Klein, J.P.; Sibilia, J.; Wachsmann, D. ERK 1/2- and JNKs-dependent Synthesis of Interleukins 6 and 8 by Fibroblast-like Synoviocytes Stimulated with Protein I/II, a Modulin from Oral Streptococci, Requires Focal Adhesion Kinase. J. Biol. Chem. 2003, 278, 27721-27728. [CrossRef]

Sample Availability: Samples of the compounds are currently not available from the authors.

Publisher's Note: MDPI stays neutral with regard to jurisdictional claims in published maps and institutional affiliations.

(C) 2020 by the authors. Licensee MDPI, Basel, Switzerland. This article is an open access article distributed under the terms and conditions of the Creative Commons Attribution (CC BY) license (http://creativecommons.org/licenses/by/4.0/). 